Progress In Electromagnetics Research M, Vol. 37, 83-93, 2014

\title{
Micro-Doppler Extraction and Analysis of the Ballistic Missile Using RDA Based on the Real Flight Scenario
}

\author{
Joo-Ho Jung ${ }^{1}$, Kyung-Tae Kim¹, Si-Ho Kim², and Sang-Hong Park ${ }^{3}$, *
}

\begin{abstract}
Micro-Doppler (MD) caused by the motion of the ballistic missile can contribute to successful recognition of the ballistic missile. Considering the real observation scenario. This paper proposes a method to derive the MD image of the ballistic missile by applying the range-Doppler algorithm (RDA) based on the real flight scenario and analyzes the factor for the real-time MD imaging. Simulation results using the flight trajectory constructed using the real target parameter demonstrate that we need a new cost function for phase adjustment and a new method for range alignment. In addition, matched-filtering needs to be performed in the baseband, and a sufficient PRF is required to prevent discontinuity of the MD image. Dechirping of MD and filtering of the random movement are also needed for a clear MD image.
\end{abstract}

\section{INTRODUCTION}

Among the various weapons used in the modern battlefield, the ballistic missile inflicts the biggest threat due to its high maneuvering speed and low radar cross section (RCS), and thus defending against the ballistic missile is a major issue. Recently, a theory has been developed to explain micro-Doppler (MD) effect caused by the micro motion of the target and applied for radar target recognition purpose $[1,2]$. In the case of the ballistic missile, three motion components - spinning, conning and nutation - cause $\mathrm{MD}$, and they can be utilized for target recognition in combination with the motion parameter $[1,3,4]$.

However, very little research has been reported on its application to the real flight scenario and the factor that needs to be considered for the real-time MD imaging. In this paper, considering the real observation scenario by a radar, we propose a method to extract an MD signature of a ballistic missile engaged in the real flight scenario by applying the range-Doppler algorithm (RDA), which is generally used to form the inverse synthetic aperture radar (ISAR) image [5-8], and analyze various factors for the real-time high quality MD image. For this purpose, we constructed the flight trajectory by using the real motion parameters of a $500 \mathrm{~km}$ range scud missile conducted a translation motion compensation (TMC). Then, the time-varying MD image was formed by applying the time-frequency transform (TFT). Various simulations were performed by using the obtained MD image to study the requirement for the real-time $\mathrm{MD}$ imaging.

Simulation results obtained by using a target composed of the point scatter demonstrate that the MD signature can be successfully constructed by using the range-Doppler algorithm. However, a new method for TMC is required for real-time high-quality MD imaging. In addition, matched-filtering (MF) in the baseband is required to form a focused image, and a sufficient PRF is needed to remove discontinuity. The reflected signal needs to be dechirped to reduce the required PRF, and a filter needs to be designed to remove the random movement.

Received 8 April 2014, Accepted 22 June 2014, Scheduled 27 June 2014

* Corresponding author: Sang-Hong Park (radar@pknu.ac.kr).

1 Department of Electrical Engineering, Pohang University of Science and Technology, Pohang, Gyungbuk, Korea. ${ }^{2}$ Agency for Defense Development, Daejeon, Korea. ${ }^{3}$ Department of Electronic Engineering, Pukyong National University, Busan, Korea. 


\section{PRINCIPLES}

\subsection{Signal Model Using the Chirp Signal}

For the radar signal, we assume the monostatic chirp waveform [9]. The transmitted signal at fast-time $t$ is given by

$$
s_{0}(t)=\exp ^{j 2 \pi\left(f_{0} t+\frac{B t^{2}}{2 \tau}\right)} \times \operatorname{rect}\left(\frac{t}{\tau}\right)
$$

where $A_{0}$ is its amplitude, $f_{0}$ the start frequency, $B$ the bandwidth, $\tau$ the pulse duration, and rect a function whose value is 1 for $t-\tau / 2 \leq t \leq t+\tau / 2$ and 0 otherwise. At the slow-time $t s$ given in the pulse repetition period $T s$, the received signal reflected from a target composed of $K$ scattering centers is as follows:

$$
g\left(t, t_{s}\right)=\sum_{k=0}^{K} A_{k} \exp ^{j 2 \pi\left(f_{0}\left(t-d_{k}\left(t_{s}\right)\right)+\frac{B\left(t-d_{k}\left(t_{s}\right)\right)^{2}}{2 \tau}\right)} \times \operatorname{rect}\left(\frac{t-d_{k}\left(t_{s}\right)}{\tau}\right),
$$

where $A_{k}$ is the amplitude of scattering center $k$ and $d_{k}\left(t_{s}\right)$ the fast-time delay between the radar and scattering center $k$ at $t_{s}$. In calculating $d_{k}\left(t_{s}\right)$, we utilize the plane wave approximation which projects the scatterer center onto the line-of-sight of the radar.

To obtain the high resolution profile, the reflected signal is matched filtered by correlating $g\left(t, t_{s}\right)$ with the stored replica of (1). Because of the computation time, MF is conducted in fast-time frequency domain by multiplying the received signal by the conjugate of the replica. Using the stationary phase approximation $[10,11]$, the fast-time frequency domain signal $G\left(f, t_{s}\right)$ of $g\left(t, t_{s}\right)$ is

$$
G\left(f, t_{s}\right)=\sum_{k=0}^{K} A_{k} \exp \left(j 2 \pi f d_{k}\left(t_{s}\right)\right) \exp \left(j 2 \pi\left(0.5 \frac{\tau}{B}\left(f-f_{0}\right)^{2}\right)\right) .
$$

The $S_{0}(f)$ of $s_{0}(t)$ can be calculated in the same way, and the resultant the signal after MF is given as follows:

$$
S_{M}\left(f, t_{s}\right)=\sum_{k=0}^{K} A_{k} \exp \left(j 2 \pi f d_{k}\left(t_{s}\right)\right) .
$$

Finally, the compressed high-resolution signal in fast time domain is given by

$$
S_{M}\left(t, t_{s}\right)=\sum_{k=1}^{K} I F T\left\{A_{k} \exp \left(j 2 \pi f d_{k}\left(t_{s}\right)\right)\right\}=\sum_{k=1}^{K} A_{k} \operatorname{sinc}\left(B\left(t-d_{k}\left(t_{s}\right)\right)\right),
$$

which is an RP of the target at slow-time $t_{s}$. MD is caused by the time-varying $d_{k}\left(t_{s}\right)$, thus the MD signature is derived by performing the TFT of the slow-time signal at $t$. Using the well-known Doppler formula [12], the MD of the scatterer $k$ at ts is given by

$$
M D_{k}\left(t_{s}\right)=-\frac{2 v_{l o s}}{\lambda}=-\frac{2}{\lambda} \frac{d d_{k}\left(t_{s}\right)}{d t_{s}},
$$

where $v_{l o s}$ is the target velocity projected onto the radar line-of-sight.

\subsection{Motion of a Ballistic Missile on a Fixed Frame}

Assuming that the ballistic missile is fixed, the motion is composed of four components: spinning, coning, nutation and vibration (Fig. 1). Spinning and coning are the rotation around an axis in a local coordinate $(x, y, z)$, and the difference is that the axis of spinning is inside the missile, and that of coning is outside. Nutation is the rotation of the target in the plane formed by the time-varying spinning axis and the stable conning axis. Vibration is the random movement of the target due to the atmospheric condition in arbitrary directions.

Spinning and conning can be explained by Rodrigues' rotation formula, which gives an efficient method to derive the rotation matrix $M$ corresponding to a rotation by an angle $\theta$ around a unit 


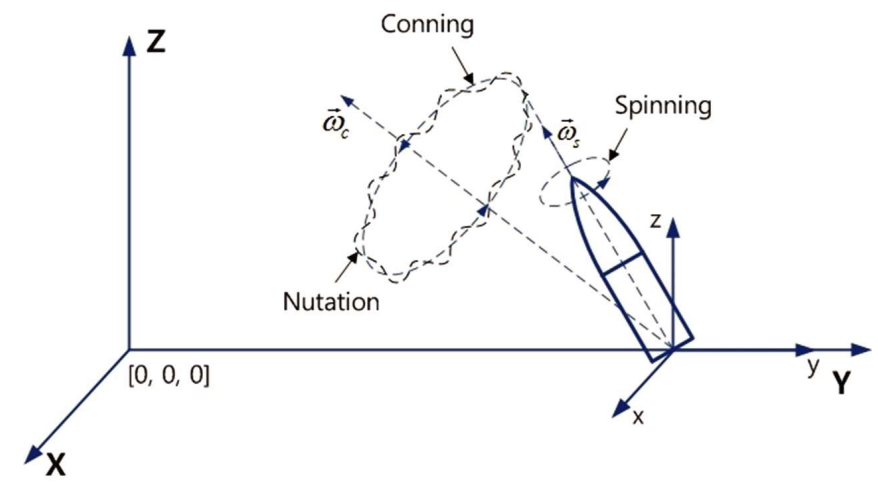

Figure 1. Motion of a ballistic missile.

vector $[1,3]$. Assuming that a vector $\vec{v}$ is rotated by $\theta$ around the an axis $\vec{k}=\left(k_{1}, k_{2}, k_{3}\right)$, the rotated vector $\vec{v}_{r o t}$ is expressed by

$$
\vec{v}_{r o t}=\left(I \cos \theta+[\vec{k}]_{x} \sin \theta+(1-\cos \theta) \vec{k} \vec{k}^{T}\right)=M \vec{v}
$$

where

$$
[\vec{k}]_{x}=\left[\begin{array}{ccc}
0 & -k_{3} & k_{2} \\
k_{2} & 0 & -k_{1} \\
-k_{2} & k_{1} & 0
\end{array}\right]
$$

For a scatter engaged in spinning and coning motion starting from $\vec{p}_{0}$, the transient position is as follows [1]:

$$
\vec{p}_{t}=\mathbf{M}_{c}\left(t_{s}\right) \mathbf{M}_{s}\left(t_{s}\right) \vec{p}_{0},
$$

where $\mathbf{M}_{c}$ and $\mathbf{M}_{s}$ are the rotation matrix constructed using the spinning velocity vector $\vec{w}_{s}$ and the coning velocity vector $\vec{w}_{c}$. Note that $\theta$ in (7) is replace by $\left|\vec{w}_{s}\right| t_{s}$ and $\left|\vec{w}_{c}\right| t_{s}$.

To model the nutation, we use the method proposed in [3], which transforms the position vector in $(x, y, z)$ into that in the new coordinate $\left(x^{\prime}, y^{\prime}, z^{\prime}\right)$ formed by the spinning and coning axes. The transformed matrix is then rotated by using a sinusoidal rotation angle, and the rotated vector is transformed back to the original coordinate. This procedure is explained by the following matrix (see [3] for the detailed procedure):

$$
\mathbf{M}_{n}\left(t_{s}\right)=\mathbf{T M}_{a}\left(t_{s}\right) \mathbf{M}^{T}
$$

where

$$
\mathbf{T}_{T}=\left[\begin{array}{lll}
\hat{x} & \hat{y} & \hat{z}
\end{array}\right], \quad \mathbf{M}_{a}=\left[\begin{array}{ccc}
\cos \theta\left(t_{s}\right) & -\sin \theta\left(t_{s}\right) & 0 \\
\sin \theta\left(t_{s}\right) & \cos \theta\left(t_{s}\right) & 0 \\
0 & 0 & 1
\end{array}\right], \quad \theta\left(t_{s}\right)=\theta_{n} \sin \omega_{n} t_{s} .
$$

$\hat{x}, \hat{y}$ and $\hat{z}$ are the unit vector of $\left(x^{\prime}, y^{\prime}, z^{\prime}\right)$ in $(x, y, z)$, and the superscript $\mathbf{T}$ is transposition. Finally, the motion of a ballistic missile engaged in spinning, coning and nutation is expressed by the simple matrix form as follows:

$$
\vec{p}_{n}=\mathbf{M}_{n}\left(t_{s}\right) \mathbf{M}_{c}\left(t_{s}\right) \mathbf{M}_{s}\left(t_{s}\right) \vec{p}_{0} .
$$

Vibration is a random motion of the target due to the atmospheric turbulence and can be modeled by the addition of the random 3D motion to the ballistic missile motion as follows:

$$
\vec{p}_{f}=\vec{p}_{n}+A \vec{n}\left(t_{s}\right)
$$

where $\vec{n}\left(t_{s}\right)$ is the unit vector in arbitrary direction at $t_{s}$ and $A$ the amplitude. 


\subsection{Construction of the Flight Trajectory}

The flight of the ballistic missile is composed of three phases: boost, midcourse and terminal. The boost phase is the portion of the flight of a ballistic missile which consumes fuel. The midcourse phase is the flight course outside the atmosphere, and the terminal phase is the flight trajectory between the reentry into the atmosphere and the point of impact.

The boost phase is modeled by solving the equation of motion in the geometry in Fig. 2. Five parameters are considered in boost phase: thrust $T_{h}$ by the propellant, velocity $V$, lift $L$, drag force $D$, and gravitational force $F_{g}=m g$, where $m$ is the weight of the ballistic missile and $g$ the gravitational acceleration (see $[13,14]$ for the definition of each parameter).

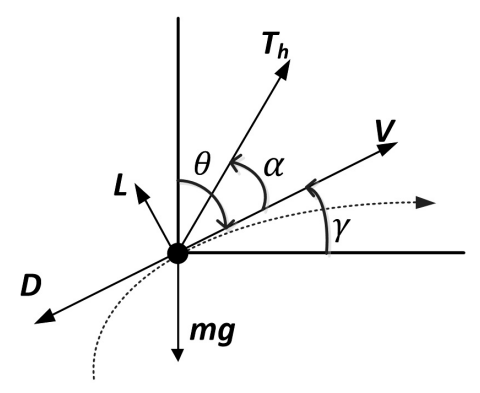

Figure 2. Geometry in boost phase.

The horizontal and vertical equations of missile motion in Fig. 2 can be expressed as follows [13]:

$$
\begin{aligned}
& \text { Horizontal }: m a=m \frac{d V}{d t_{s}}=T_{h} \cos \alpha-D-m g \sin \gamma \\
& \text { Verticall }: m V \omega=m V \frac{d \gamma}{d t_{s}}=T_{h} \cos \alpha+L-m g \cos \gamma
\end{aligned}
$$

Assuming $\alpha=0$ and $L=0$, the distance of flight is obtained by integrating $V$ in the horizontal equation, and the angle at the calculated distance is obtained by $\gamma$ in the vertical equation. When the pitch program $P\left(t_{s}\right)$ following the vertical ascent is conducted to achieve the $\gamma$ for the maximum range and the angular acceleration, angular acceleration is given by

$$
\dot{\gamma}=P\left(t_{s}\right)+\frac{d \gamma}{d t_{s}}=P\left(t_{s}\right)-\frac{g\left(t_{s}\right) \cos \gamma\left(t_{s}\right)}{V\left(t_{s}\right)},
$$

In the midcourse and terminal phases, it can be assumed that the ballistic missile is launched at an initial velocity $V_{0}$ and shooting angle $\gamma$. The ballistic undergoes the drag force in the opposite direction given as follows:

$$
a=\frac{\pi C_{D}\left(t_{s}\right) \rho\left(t_{s}\right) V^{2} d^{2}}{8 m} .
$$

Using $V=V_{0}-a t_{s}, a$ is derived by solving the second order equation as follows:

$$
a=\left(\frac{V_{0}}{t_{s}}+\frac{4 m}{\pi C_{D}\left(t_{s}\right) \rho\left(t_{s}\right) V^{2} d^{2}}\right)+\sqrt{\left(\frac{V_{0}}{t_{s}}+\frac{4 m}{\pi C_{D}\left(t_{s}\right) \rho\left(t_{s}\right) V^{2} d^{2}}\right)-\frac{V_{0}^{2}}{t_{s}^{2}}},
$$

where $C_{D}\left(t_{s}\right)$ and $\rho\left(t_{s}\right)$ are the drag coefficient and the atmospheric density at $t_{s} . d$ is the diameter of the missile (see $[13,14]$ for the parameters). As a result, the horizontal and vertical positions of the missile are as follows:

$$
x=\left(V_{0} \cos \gamma t_{s}-\frac{1}{2} a \cos \gamma t_{s}^{2}\right), \quad y=V_{0} \sin \gamma t_{s}-\frac{1}{2}\left(a \sin \gamma-g\left(t_{s}\right)\right) t_{s}^{2} .
$$




\subsection{Construction of the Micro-Motion on the Flight Trajectory}

Because the micro-motion in Subsection 2.2 was developed on the basis of the target rotating around the fixed coning axis, it is required to transform to the motion of a target flying along the trajectory. In a real flight, the coning axis changes, and as a result, the MD changes considerably.

The transform matrix to transform the fixed target motion to the moving target motion is computed by using the transformation of the unit vector of the fixed coning axis $\left(\vec{v}_{1}\right)$ to that of the real-time coning axis $\left(\vec{v}_{2}\right)$ (Fig. 3 ). Transformation is completed by rotating $\vec{v}_{1}$ counterclockwise by $\theta_{x z 1}$ about $z$ axis, clockwise by $\theta_{y z}$ in $y$ - $z$ plane and then clockwise by $\theta_{x z 2}$ about $z$ axis. The corresponding rotation matrices are as follows:

$$
\mathbf{M}_{z 1}=\left[\begin{array}{ccc}
\cos \left(\theta_{x z 1}\right) & -\sin \left(\theta_{x z 1}\right) & 0 \\
\sin \left(\theta_{x z 1}\right) & \cos \left(\theta_{x z 1}\right) & 0 \\
0 & 0 & 1
\end{array}\right], \quad \mathbf{M}_{x}=\left[\begin{array}{ccc}
1 & 0 & 0 \\
0 & \cos \left(\theta_{y z}\right) & -\sin \left(\theta_{y z}\right) \\
0 & \sin \left(\theta_{y z}\right) & \cos \left(\theta_{y z}\right)
\end{array}\right], \quad \mathbf{M}_{z 2}=\left[\begin{array}{ccc}
\cos \left(\theta_{x z 2}\right) & -\sin \left(\theta_{x z 2}\right) & 0 \\
\sin \left(\theta_{x z 2}\right) & \cos \left(\theta_{x z 2}\right) & 0 \\
0 & 0 & 1
\end{array}\right] .
$$

$\vec{p}_{v}(t)$ in the local coordinate is conducted as follows:

$$
\vec{p}_{t r}\left(t_{s}\right)=\left(\mathbf{M}_{z 2}\right)^{-1} \times\left(\mathbf{M}_{x}\right)^{-1} \times \mathbf{M}_{z 1} \vec{p}_{n}\left(t_{s}\right)+\left(\mathbf{M}_{z 2}\right)^{-1} \times\left(\mathbf{M}_{x}\right)^{-1} \times \mathbf{M}_{z 1} \vec{n}_{s}\left(t_{s}\right)+\vec{r}_{0}\left(t_{s}\right),
$$

where $\vec{r}_{0}\left(t_{s}\right)$ is the position of the origin of the local coordinate in the global coordinate.

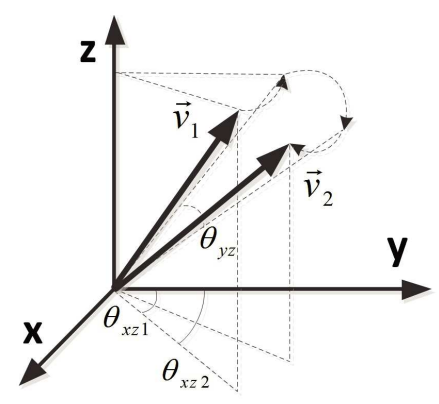

Figure 3. Two vectors to obtain transformation matrix.

\subsection{TMC}

TMC is conducted to remove the effect of the translational motion of the missile. TMC is composed of two steps; the range alignment (RA) to position an identical scatter at the identical range bin and the phase adjustment (PA) to remove residual phase error. In RA, the method that finds the shift minimizing the one dimensional (1D) entropy is widely used [15-17]. The $1 \mathrm{D}$ entropy between two RPs is defined by as follows

$$
H_{G_{m}: G_{m+1}}=-\sum_{0}^{N-1} G(n) \ln G(n)
$$

where $G(n)$ is the sum of the two RPs. In PA, the phase error that minimizes the two dimensional (2D) entropy is found [16]. The radar signal after PA can be denoted by

$$
S_{M P}(m, n)=S_{M A}(m, n) \exp \left[-j \phi_{c}(m)\right]
$$

where $S_{M P}$ is the radar signal after RA and $\phi_{c}(m)$ the phase error of RP $\mathrm{m}$. The PA finds the phase error that minimizes the following $2 \mathrm{D}$ entropy:

$$
H=-\sum_{m=0}^{M-1} \sum_{n=0}^{N-1} I(m, n) \ln I(m, n)
$$

where $I$ is the normalized ISAR image derived by fast Fourier transforming the cross-range signal for each $n$ in (22) (see [15-17]). 


\subsection{Overall Procedure}

The overall procedure to extract MD of the ballistic missile is composed of five steps (Fig. 4). The first step is to model the radar signal given in subsections from 2.1 to 2.4. The second and third steps are RA and PA. The fourth step selects the range bin that contains the radar signal of the ballistic missile. In this paper, we use the range bin containing the maximum energy. Finally, the TFT is conducted to express the time-varying MD of the ballistic missile in TF domain. Among several TFT methods, we use the short-time Fourier transform $[18,19]$.

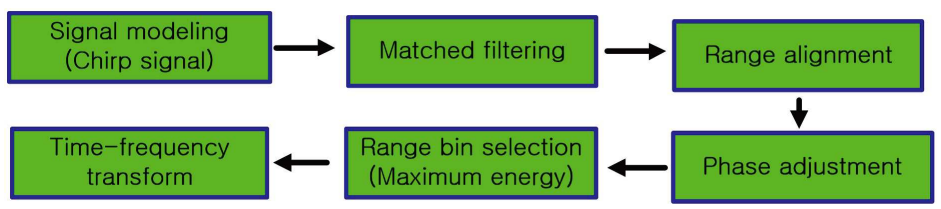

Figure 4. Signal processing procedure.

\section{SIMULATION RESULTS}

\subsection{Simulation Set Up}

In simulations, we used the warhead of the ballistic missile composed of the isotropic point scatterer spinning around the $z$-axis (Fig. 5(a)), and an observation was conducted for $2 \mathrm{~s}$ for $236 \leq t s \leq 238$. The initial attitude of the target and the spinning axis were determined by sequentially rotating the target by $-10^{\circ}$ around $z$-axis, $-15^{\circ}$ around $x$-axis and $-5^{\circ}$ around $z$-axis (Fig. $5(\mathrm{~b})$ ). The ballistic missile was assumed to have $\left|\vec{\omega}_{s}\right|=7 \pi \mathrm{rad} / \mathrm{s}, \omega_{c} \mid=2 \pi$, and $\omega_{n} \mid=2 \pi$ with $\theta_{n}=2^{\circ}$. A $500 \mathrm{~km}$ range SCUD-C missile with the total weight of $7200=5000$ (payload) +1130 (structure) +870 (propellant) $\mathrm{Kg}$ was simulated by using the missile parameters in Table 1 (see [14]).

For the radar system, we used a monostatic chirp radar with pulse repetition frequency $(\mathrm{PRF})$ $=6 \mathrm{kHz}$, center frequency $=10 \mathrm{GHz}$, bandwidth $B=50 \mathrm{MHz}$, and the pulse width $=30 \mu \mathrm{s}$. The radar was located at $300 \mathrm{~km}$ from the origin in the plane formed by the trajectory.

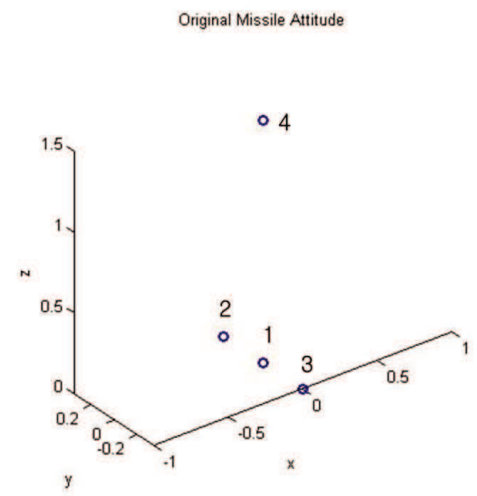

(a)

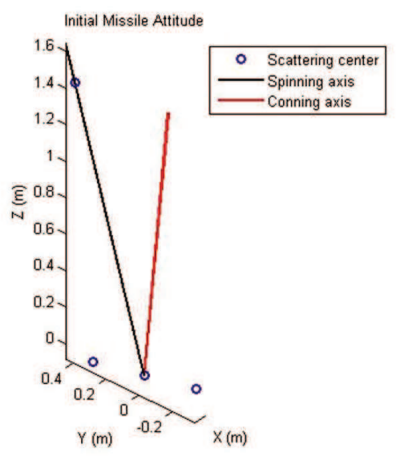

(b)

Figure 5. Attitude of ballistic missile. (a) Original attitude. (b) Initial attitude.

Table 1. Parameters for missile.

\begin{tabular}{|c|c|c|c|c|c|}
\hline Burnout time & $87.5 \mathrm{~s}$ & $d$ & $1 \mathrm{~m}$ & $T_{h}$ & $134 \mathrm{KN}$ \\
\hline Vertical ascent time & $7 \mathrm{~s}$ & Pitch program time & $14 \mathrm{sec}$ & Pitch program angle & $20^{\circ}$ \\
\hline
\end{tabular}




\subsection{Simulation Result of the Flight Trajectory}

To achieve $500 \mathrm{~km}$ range, the pitch program was designed such that the missile linearly turned by $b \%$ of the total turning angle for each of the initial and the final $c \%$ of the pitch program period and $(100-2 b) \%$ for $(1-c) \%$ (Fig. 6(a)). The $b=0.005$ and $c=0.2$ that provided $500 \mathrm{~km}$ range were selected. The missile successfully landed at $500 \mathrm{~km}$ from the origin (Fig. 6(b)).

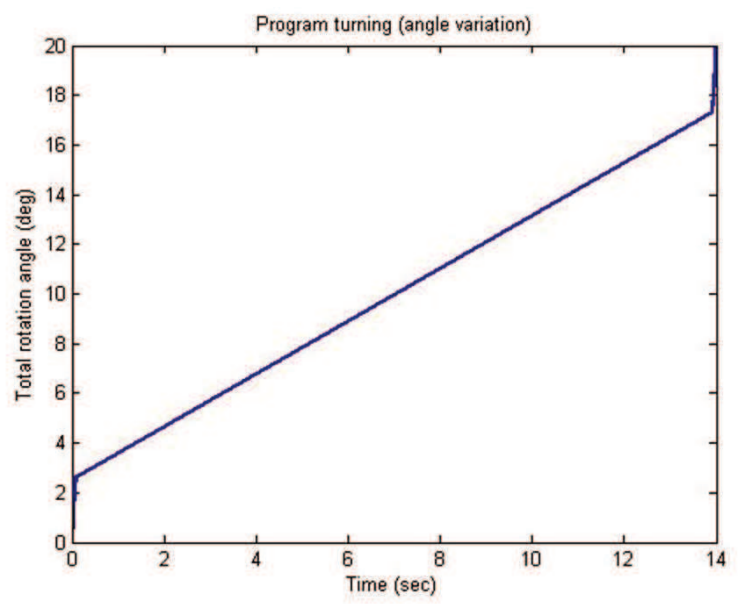

(a)

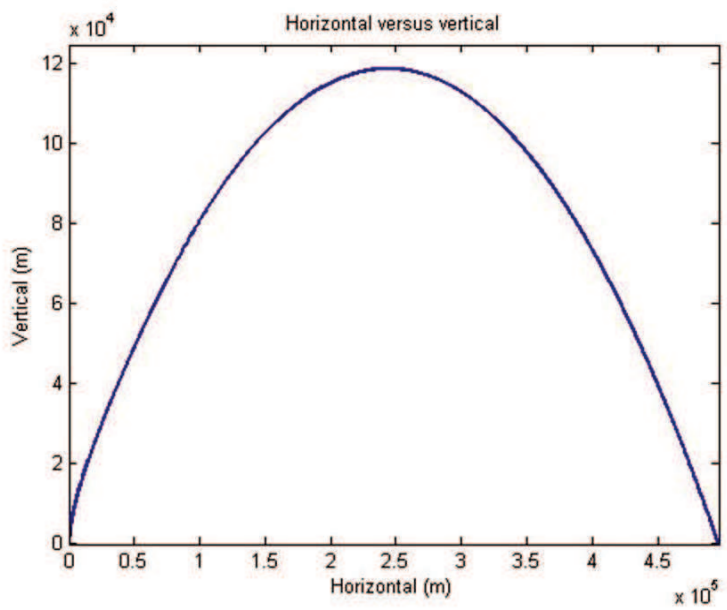

(b)

Figure 6. Simulation result for the trajectory. (a) Pitch program. (b) Total trajectory.

\subsection{MD of the Moving Ballistic Missile after Conventional RDA}

The collected signal with $\vec{n}(t)=0$ was processed, and the initial MD was set to zero by subtracting the Doppler frequency corresponding to the initial speed. Because of the missile movement, the history of the RPs collected was represented by a curve (Fig. 7(a)). RPs were well aligned by RA (Fig. 7(b)), and the radar signal in the range bin with the maximum energy was successfully extracted (Fig. 7(c)).

The MD image derived from TFT and that calculated by using the known missile speed were significantly different (Fig. 8), and that without PA was identical to the calculated image. This is because the 2D entropy of the ISAR image is the degree of the image focus, thus decreases as the pixels in ISAR image are concentrated in a narrow region. Therefore, PA using $2 \mathrm{D}$ entropy gives an adverse effect to MD image, and a proper cost function is required to yield the clear MD image.

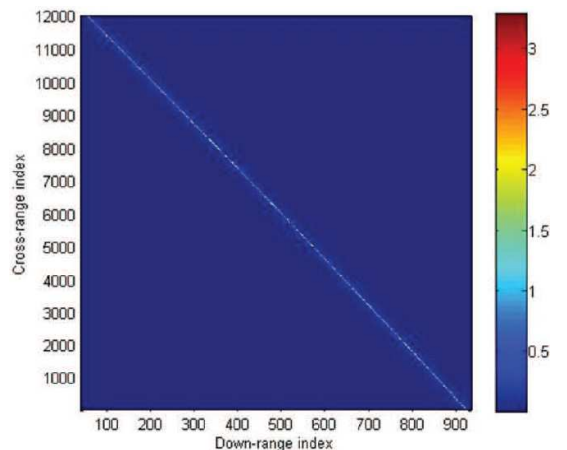

(a)

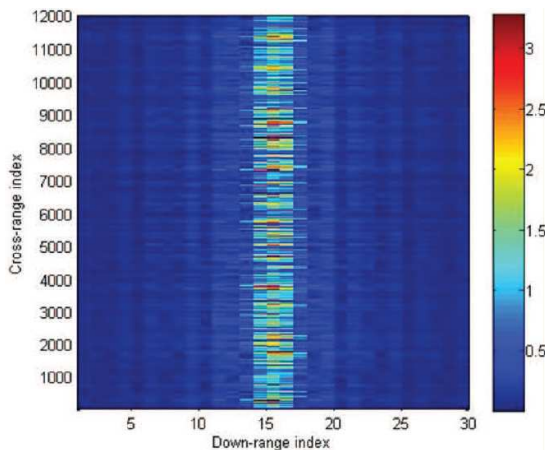

(b)

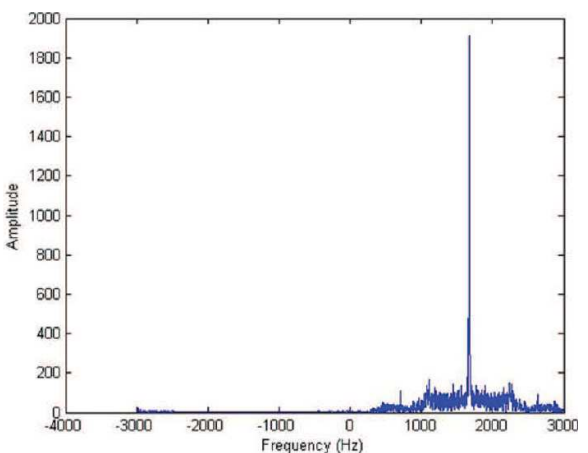

(c)

Figure 7. Simulation result using a moving target. (a) RP history. (b) Aligned RP. (c) Signal in the range bin $n_{0}$. 


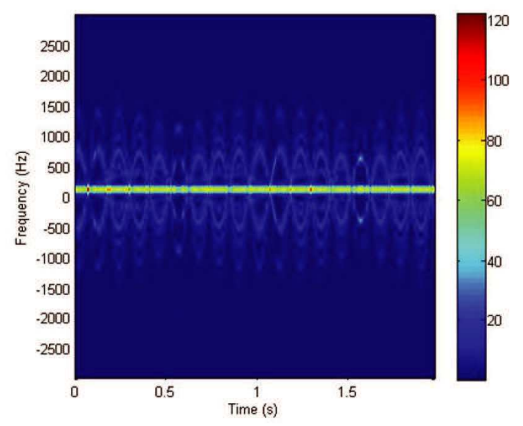

(a)

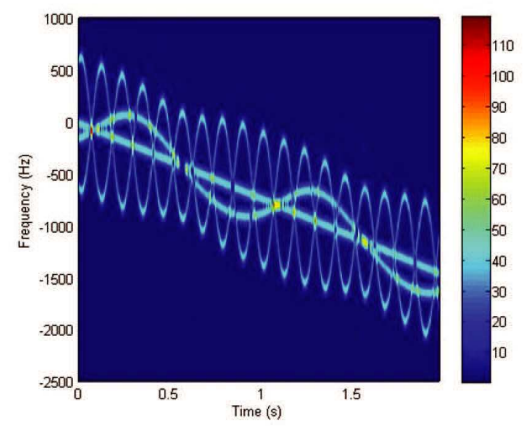

(b)

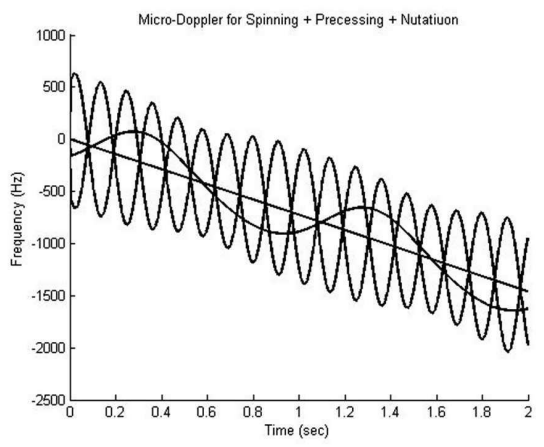

(c)

Figure 8. Comparison of MD images. (a) With PA. (b) Without PA. (c) Calculated.

\subsection{Analysis on Various Factors}

\subsubsection{MF in Carrier and Basebands}

The mathematical formulation for the MF in (5) allows MF to be carried out in the carrier band; however, a small sampling error may break the coherency of the radar signal in a range bin, and this can seriously damage the MD image (Fig. 9). Therefore, the baseband conversion of the reflected signal is an indispensable procedure for the focused MD image.

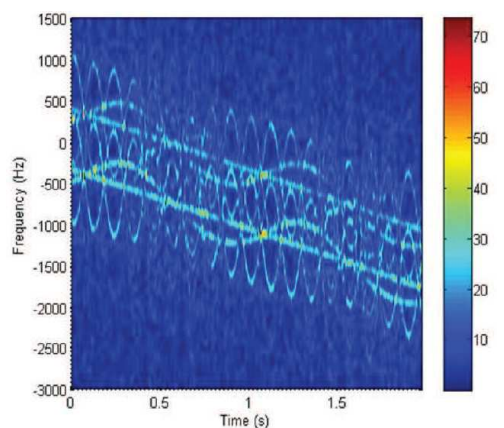

(a)

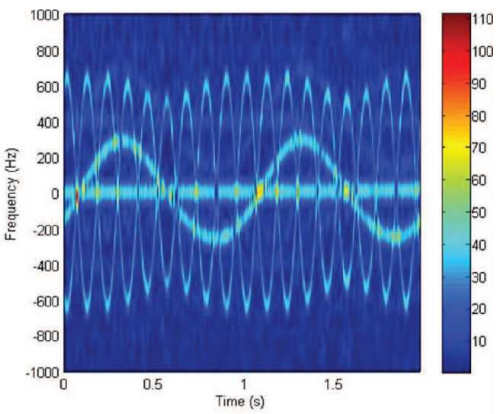

(b)

Figure 9. MD images obtained by carrier- and base-band MFs ( $B=90 \mathrm{MHz}$ ). (a) Carrier-band MF. (b) Baseband MF,

\subsubsection{Upsampling of the Trajectory}

In simulating the flight trajectory, PRF can be reduced to save the computation time, and the downsampled trajectory can be interpolated. However, discontinuity of MD can occur in the MD image because of the interpolation error (Fig. 10). Thus, high PRF is required in spite of the increased computation time.

\subsubsection{Dechirping of $M D$}

The missile after boost phase is accelerated downward because of the gravity. This acceleration widens the bandwidth by tilting the MD downward, and thus high PRF is required for long-time observation to avoid aliasing in the frequency domain (Fig. 11(a)). Therefore, dechirping by measuring the realtime velocity onto the radar line-of-sight is required before TFT. The MD image obtained by using the dechirped signal is flat, and $2 \mathrm{kHz}$ PRF is sufficient to yield the alias-free image (Fig. 11(b)). 


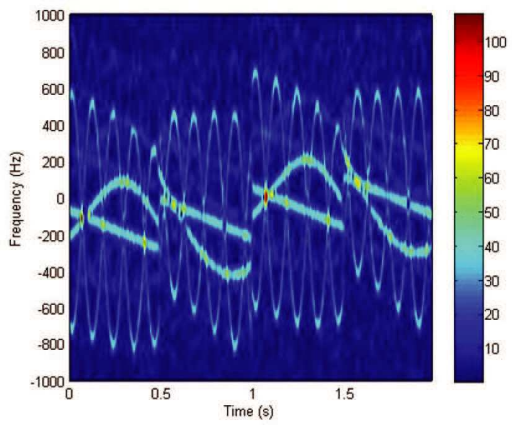

Figure 10. MD images of the up-sampling trajectory (down-sampling at $2 \mathrm{~Hz}$ ).

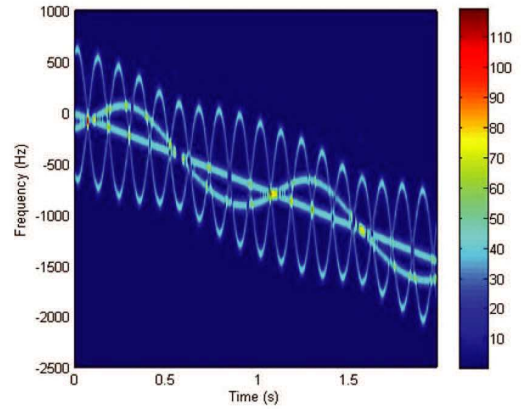

(a)

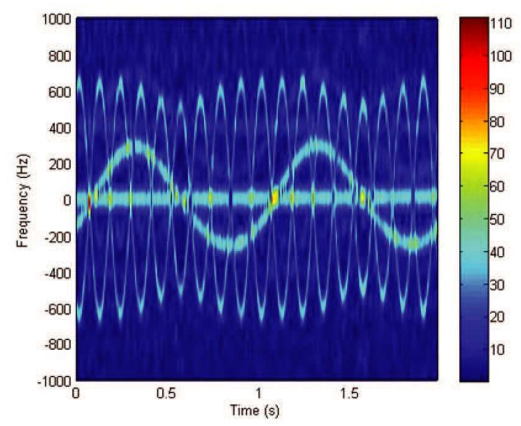

(b)

Figure 11. MD images before and after dechirping $(B=50 \mathrm{MHz})$. (a) Before dechirping. (b) After dechirping.

\subsubsection{Bandwidth}

High-resolution RPs can contribute to the increased classification ratio; however, the increased number of RPs may yield poorly aligned RPs. Thus, we studied the effect of $B$ in extracting the MD of the missile. The two images obtained by using $B=50 \mathrm{MHz}$ and $200 \mathrm{MHz}$ were not considerably different (Fig. 12). However, the 2D entropy increased owing to the poor alignment of the increased number of range bins at higher $B$. Therefore, a new form of cost function is required to extract MD image at wide bandwidth.

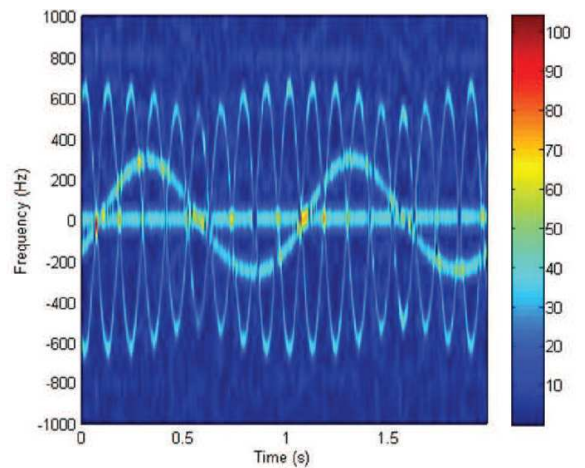

(a)

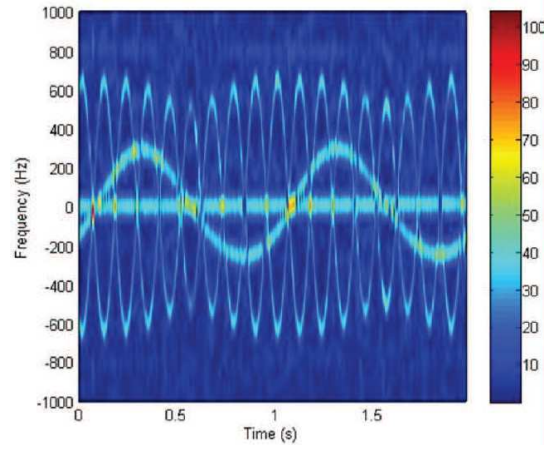

(b)

Figure 12. Results of RA and MD images for two $B$ s. (a) MD image ( $B=50 \mathrm{MHz}$, Ent $=12.0832$ ). (b) MD image $(B=200 \mathrm{MHz}, 12.1196)$ 


\subsubsection{Time for $R A$}

We studied the time for RA for various Bs and observation time for the real-time MD imaging. The time for RA was measured by using the entropy minimization given in [15]. RA was more dependent on the observation period, and the computation time was directly proportional to the bandwidth because of the increased number of range bines (Table 2). The huge computation time demonstrates that a fast method of RA should be developed for the real-time MD imaging.

Table 2. Time for RA for various $B$ s and observation time.

\begin{tabular}{ccccccc}
\multirow{2}{*}{$B(\mathrm{MHz})$} & \multicolumn{6}{c}{ Observation time $(\mathrm{s})$} \\
\cline { 2 - 7 } & 0.5 & 1 & 1.5 & 2 & 2.5 & 3 \\
\hline 20 & 0.53 & 2.08 & 5.24 & 9.84 & 16.94 & 25.98 \\
40 & 0.76 & 3.4 & 9.18 & 18.54 & 33.31 & 52.66 \\
60 & 0.94 & 4.75 & 13.4 & 28 & 51.12 & 82.75 \\
80 & 1.14 & 6.09 & 17.56 & 37.66 & 68.9 & 112.99 \\
100 & 1.33 & 7.59 & 22.26 & 47.97 & 89.27 & 148.15
\end{tabular}

\subsubsection{Random Movement}

The effect of the random movement by the turbulence on the MD image was also studied. Three MD images were compared by using $A=0.001,0.003$ and 0.005 with $B=50 \mathrm{MHz}$ (Fig. 13). The degree of MD distortion was proportional to $A$ (Fig. 13), and thus, an algorithm to estimate and filter out the $3 \mathrm{D}$ random motion is required for the clear MD image.

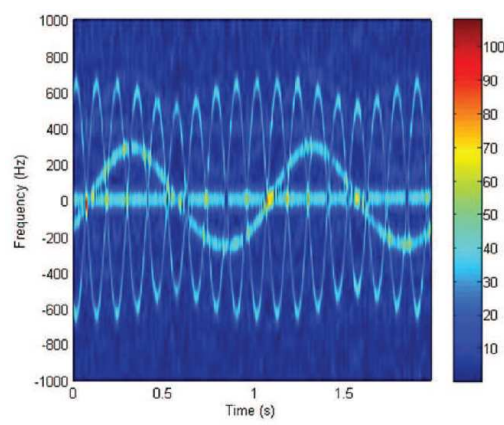

(a)

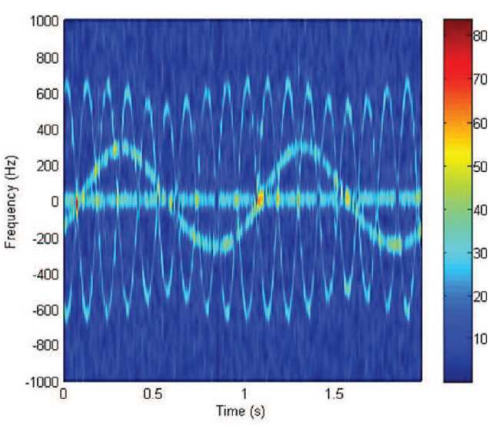

(b)

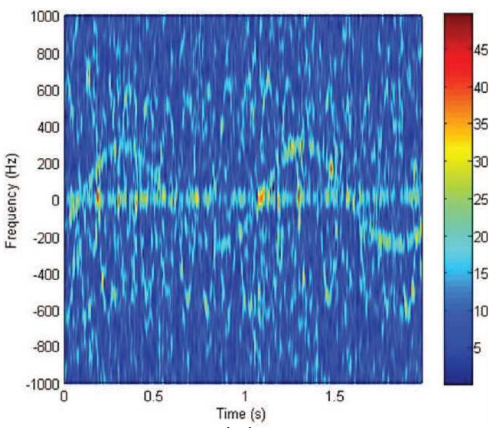

(c)

Figure 13. MD images for three $A \mathrm{~s}(B=50 \mathrm{MHz})$. (a) $A=0.001$. (b) $A=0.003$. (c) $A=0.005$.

\section{CONCLUSION}

In this paper, we propose a method to extract the MD signature by using RDA and the real observation scenario by a radar. Various simulations were conducted by using the model composed of the ideal point scatterers engaged in micro-motion, and the requirement for RDA was analyzed to obtain the real-time high-quality MD image.

The MD image after PA was significantly different from the ideal MD. Therefore, a new cost function was needed. In addition, a new method for RA was required to align RPs regardless of the number of range bins with a small computation time. MF in the baseband was necessary to maintain the coherency and a sufficient PRF was needed to remove discontinuity in simulation. In addition, dechirping of the reflected signal was needed to reduce the required PRF. A filter that removes the random movement was also required to remove the $3 \mathrm{D}$ random motion.

However, it should be pointed out that in this study, performance evaluation of the proposed scheme has only been based on the ideal point scatterers and the simulated flight trajectory of the scud missile. 
Thus, the performance verification in Section 3 is limited in this regard. Therefore, we currently are planning to demonstrate our analysis results obtained by using MD images from real radar data; the associated results will be presented in a journal in the near future.

\section{ACKNOWLEDGMENT}

This work was supported by the STRL (Sensor Target Recognition Laboratory) program of Defense Acquisition Program Administration and Agency for Defense Development.

\section{REFERENCES}

1. Chen, V. C., The Micro-Doppler Effect in Radar, Artech House, 2011.

2. Chen, V. C., F. Li, S. Ho, and H. Wechsler, "Micro-Doppler effect in radar," IEEE Trans. Aerospace Electron Syst., Vol. 42, No. 1, 2-21, Oct. 1996.

3. Gao, H., L. Xie, S. Wen, and Y. Kuang, "Micro-Doppler signature extraction from ballistic target with micro-motions," IEEE Trans. Aerospace Electron Syst., Vol. 46, No. 4, 1969-1982, Oct. 2010.

4. Liu, L., X. Du, M. Ghogho, W. Hu, and D. McLernon, "Precession missile feature extraction using sparse component analysis of radar measurements," EURASIP J. Adv. Sig. Pr., Vol. 2012, No. 24, 1-10, Feb. 2012.

5. Park, S.-H., J.-H. Lee, and K.-T. Kim, "Performance analysis of the scenario-based construction method for real target ISAR recognition," Progress In Electromagnetics Research, Vol. 129, 137151, 2012.

6. Park, J.-H. and N. H. Myung, "Enhanced and efficient ISAR image focusing using the discrete Gabor representation in an oversampling scheme," Progress In Electromagnetics Research, Vol. 138, 227-244, 2013.

7. Naqvi, A. and H. Ling, "Time-frequency and ISAR characteristics of wind turbines with higher order motions," Progress In Electromagnetics Research, Vol. 143, 331-347, 2013.

8. Choi, I.-O., J.-H. Jung, S.-H. Kim, K.-T. Kim, and S.-H. Park, "Classification of targets improved by fusion of the range profile and the inverse synthetic aperture radar image," Progress In Electromagnetics Research, Vol. 144, 23-31, 2013.

9. Mahafza, B., MATLAB Simulations for Radar Systems Design Using MATLAB, Chapter 7, Champman \& Hall/CRC Press LLC, Jan. 2000.

10. Soumekh, M., Synthetic Aperturer Radar Signal Processing with MATLAB Algorithms, John Wiley \& Sons, Inc., 1999.

11. Carrara, W. G., R. S. Goodman, and R. M. Majewski, Spotlight Synthetic Aperture Radar Signal Processing Algorithms, Artech House, 1995.

12. Skolnik, M. I., Introduction to Radar Systems, 3rd Edition, McGraw-Hill Companies, Inc., 2001.

13. Hale, F. J., Introduction to Space Flight, Prentice Hall, 1993.

14. Kim, N.-J., "A study on the analysis of the flight trajectory characteristics for ballistic missiles," M.S. Thesis, Korea National Defense University, Seoul, 1999.

15. Li, X., G. Liu, and J. Ni, "Autofocusing of ISAR images based on entropy minimization," IEEE Trans. Aerospace Electron Syst., Vol. 35, No. 4, 1240-1251, Oct. 1999.

16. Jung, H. R., H. T. Ki, and K. T. Kim, "Application of subarray averaging and entropy minimization algorithm to stepped-frequency ISAR autofocus," IEEE Trans. Antennas Propag., Vol. 56, No. 4, 1144-1154, 2008.

17. Wang, J., X. Liu, and Z. Zhou, "Minimum-entropy phase adjustment for ISAR," IEE Proc. of Radar, Sonar and Nav., Vol. 151, No. 4, 203-209, Aug. 2004.

18. Qian, S., Introduction to Time-frequency and Wavelet Transforms, Prentice Hall, 2002.

19. Chen, V. C. and H. Ling, Time-frequency Transforms for Radar Imaging and Signal Analysis, Artech House, 2002. 